\title{
5. Variability of Pharmacokinetic Drug-drug Interactions and Its Consequences
}

\author{
Walter E. Haefeli*
}

An inherent risk of combination therapy is the occurrence of both beneficial and adverse drug-drug interactions. They may increase effectiveness but also convey adverse drug reactions and thus limit the safety of pharmacotherapy. Similar to many regular drug effects that are highly variable between individuals ${ }^{1)}$ and do not occur in all treated patients (nonresponse), also drug interactions are highly variable. Indeed, the extent to which drugs interact is not a constant, rather is it modulated by a number of factors and their consideration in daily practice is essential to limit variability and thus risk.

A majority of drug effects is concentration-dependent and interactions modifying drug concentrations (pharmacokinetic interactions) are too. Therefore, dose, route of administration, and factors modulating the slope of the dose-concentration relationship (Figure) will also modify (and cause) variability of an interaction. As an example, administration of increasing doses of the CYP2D6 inhibitor sertraline in patients with depression progressively inhibited oxidation of the CYP2D6 substrate dextromethorphan and within a range of therapeutic sertraline doses $(50-150 \mathrm{mg} / \mathrm{d})$ CYP2D6 activity was dosedependently reduced ${ }^{2)}$. Hence, even within the same patient the result of an interaction can considerably vary. Another pertinent example of the concentrationdependency of an interaction is the transporter interaction between ciclosporin $\mathrm{A}$ and simvastatin that caused negligible changes in simvastatin exposure at low ciclosporin concentrations whereas at higher concentrations simvastatin exposure more than doubled in individual patients $^{3)}$. If doses of the statin were reduced adverse events did not occur while lipid-lowering effects persisted supporting the need of maintaining concentrations within a therapeutic range ${ }^{4)}$.

\footnotetext{
* Department of Internal Medicine VI, Clinical Pharmacology and Pharmacoepidemiology, University of Heidelberg Im Neuenheimer Feld 410, 69120 Heidelberg, Germany
}

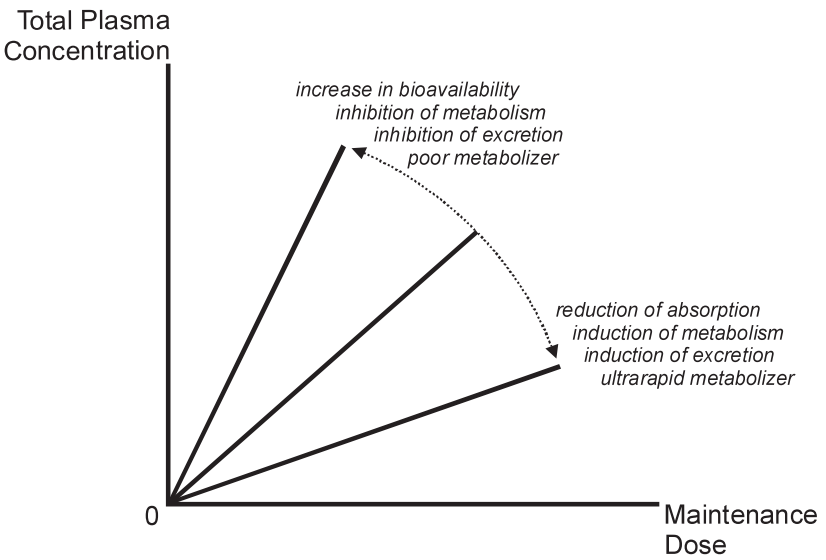

Fig. Factors affecting the relationship between administered dose and plasma concentration. Many of them are modulated by co-medication (drug-drug interaction).

However, just as expected as the concentrationdependency of pharmacokinetic drug interactions is the fact that inhibition will reach a maximum and that accumulation beyond certain levels will not further augment the interaction. Dose-dependency of an interaction is well known in antiretroviral therapy. In these patients exposure to protease inhibitors is usually boosted by small amounts ritonavir. When combined with ritonavir, indinavir exposure will increase up to a ritonavir dose of $200 \mathrm{mg}$ whereas increases to $400 \mathrm{mg}$ ritonavir had no additional effect stressing the fact that plateau effects of the interaction were reached ${ }^{5}$.

Drug interactions may also depend on the route of administration of an interaction partner. As an example, oral administration of the CYP-and transporter-inducing agent rifampin may decrease exposure with oral verapamil to $2-3 \%$ or oral propafenone to $\sim$ one forth and therefore abolish the effects of the antiarrhythmics ${ }^{6,7)}$. Because this interaction mainly occurs in the gut wall it has no effect on hepatic verapamil or propafenone clearance and, therefore, no interaction with intravenous 
verapamil or propafenone is observed ${ }^{6,7)}$.

However, even with a given dose and route of administration interactions will not occur in all patients. As an example two-week exposure with the CYP3A4 inducer St. John's wort decreases simvastatin mean AUC by half ${ }^{8)}$ albeit not in all patients. Indeed, in two of eight subjects pharmacokinetic changes were minimal, i.e. within bioequivalence margins, whereas in others statin exposure was reduced to less than $15 \%{ }^{8)}$. Similar variability was observed after combining St. John's wort with the CYP3A4 substrate midazolam ${ }^{9}$. If variability of an interaction is so large, it may be dangerous to treat all patients with a certain drug combination alike. Indeed, uniformly doubling the simvastatin dose under these conditions would result in therapeutic levels in only a small fraction of these patients.

In addition to the concentration of an inhibitor also baseline activity of the interaction target (e.g. enzyme) will affect the interaction result. If an enzyme is already inhibited by another inhibitor addition of a second inhibitor either does not further impair metabolism ${ }^{2}$ or may lead to less than additive increases in exposure ${ }^{10}$. Hence, individual factors additionally affecting metabolic capacity such as genetic variants leading to a poor metabolizer status will alter the extent of an interaction. As expected, in CYP2D6 poor metabolizers administration of a CYP2D6 inhibitor (quinidine) had no impact on the metabolic clearance of the CYP2D6 substrate propafenone ${ }^{11}$. The interplay between metabolic inhibition and enzyme inhibitors may become even more complex if several pathways are responsible for elimination of large fractions of a compound. The antifungal voriconazole is mainly eliminated by CYP3A4 and CYP2C19 of which CYP2C19 is polymorphic and responsible for $49 \%$ of pharmacokinetic variance $^{12)}$. If either pathway is inactive, i.e. if the patient is a poor metabolizer of CYP2C19 or if CYP3A4 is inhibited by a potent inhibitor (e.g. ritonavir ${ }^{13)}$ ), mild to moderate changes in clearance will occur. If, however, ritonavir is concurrently given to a CYP2C19 poor metabolizer elimination of the antifungal is abolished and exposure is 17-fold higher than in extensive metabolizers receiving this combination ${ }^{13)}$.

Inhibition of an enzyme may also vary with time i.e. within a dosing interval depending on the fluctuations (i.e. pharmacokinetics) of the inhibitor and of potential active metabolites ${ }^{14)}$. Moreover, in the case of mechanism-based inhibition gradual inactivation of the target enzyme by the inhibitor may occur. For this reason CYP3A4 activity decreases over the first 4 days of erythromycin administration although steady state is reached much earlier ${ }^{15)}$. Substantial temporal fluctuations may also be observed if drugs with opposing effects on clearance (e.g. an enzyme inducer and inhibitor) are simultaneously discontinued ${ }^{16}$ or if drugs with both properties (e.g. rifampin, isoniazid) are discontinued ${ }^{17,18)}$. If the half-life of the inhibitor is short it is likely that inhibition more rapidly disappears than induction, which takes 1 week to resolve ${ }^{9)}$. Therefore, few days after discontinuation induction may prevail leading to substantial transient increases in clearance and thus even larger dose requirements of the substrate than during administration of the inducer ${ }^{17,18)}$.

\section{Conclusion}

Substantial published evidence indicates that drug-drug interactions will not uniformly emerge in all patients receiving a particular combination and even within a given patient the extent of interaction may vary. Many of the causes for variability are predictable and should thus be considered in daily practice to identify patients on particularly dangerous combinations. Such information may also help reducing over-alerting in electronic systems.

\section{References}

1) Levy G, et al. Clin Pharmacol Ther $1994 ; 56: 1-8$.

2) Sproule BA, et al. J Clin Psychopharmacol $1997 ; 17: 102-6$.

3) Arnadottir M, et al. Nephron $1993 ; 65: 410-3$.

4) Imamura R, et al. Clin Transplant $2005 ; 19: 616-21$.

5) Saah AJ, et al. Antimicrob Agents Chemother $2001 ; 45: 2710-5$.

6) Dilger K, et al. Phamracogenetics $1999 ; 9$ : 551-9.

7) Fromm MF, et al. Hepatology $1996 ; 24: 796-801$.

8) Sugimoto K, et al. Clin Pharmacol Ther $2001 ; 70: 518-24$.

9) Imai H, et al. Br J Clin Pharmacol 2007 ; 65 : 701-7.

10) Loi CM, et al. J Pharmacol Exp Ther 1997 ; $280: 627-37$.

11) Funck-Brentano C, et al. Br J Clin Pharmacol 1989 ; 27 : 435-44.

12) Weiss J, et al. J Clin Pharmacol 2009 ; 49 : 196-204.

13) Mikus G, et al. Clin Pharmacol Ther $2006 ; 80: 126-35$.

14) Templeton IE, et al. Clin Pharmacol Ther $2008 ; 83: 77-85$.

15) Okudaira T, et al. J Clin Pharmacol 2007 ; 47 : 871-6.

16) Lam JL, et al. J Pharmacol Exp Ther 2006 ; $319: 864-70$.

17) Bidstrup TB, et al. Eur J Clin Pharmacol 2004 ; 60 : 109-14.

18) Zand R, et al. Clin Pharmacol Ther $1993 ; 54: 142-9$. 